\title{
Development and Validation of a Nomogram for Determining Patients Requiring Prolonged Postanesthesia Care Unit Length of Stay After Laparoscopic Cholecystectomy
}

\section{Bingbing Cao}

Sun Yat-sen memorial hospital

\section{Li Li}

Sun Yat-sen memorial hospital

\section{Xiangfei Su}

Sun Yat-sen memorial hospital

Jianfeng Zeng

Sun Yat-sen memorial hospital

Guo weibing ( $\nabla$ weibingguo@126.com )

Sun Yat-sen memorial hospital

\section{Research article}

Keywords: Anesthesia, Laparoscopic cholecystectomy, Postanesthesia care unit, Length of stay, Nomogram

Posted Date: August 7th, 2020

DOI: https://doi.org/10.21203/rs.3.rs-48856/v1

License: (c) (i) This work is licensed under a Creative Commons Attribution 4.0 International License.

Read Full License

Version of Record: A version of this preprint was published at Annals of Palliative Medicine on May 1st, 2021. See the published version at https://doi.org/10.21037/apm-20-2182. 


\section{Abstract}

Background: Laparoscopic Cholecystectomy (LC) is a common surgical procedure for managing gallbladder disease. Prolonged length of stay (LOS) in the postanesthesia care unit (PACU) may lead to overcrowding and a decline in medical resource utilization. In this work, we aimed to develop and validate a predictive nomogram for identifying patients who require prolonged PACU LOS.

Methods: Data from 913 patients undergoing LC at a single institution in China between 2018 and 2019 were collected, and grouped into a training set (cases during 2018) and a test set (cases during 2019). Using the least absolute shrinkage and selection operator regression model, the optimal feature was selected, and multivariable logistic regression analysis was used to build the prolonged PACU LOS risk model. The $C$-index, calibration plot, and decision curve analysis were used in assessing the model calibration, discrimination, and clinical application value, respectively. For external validation, the test set data was evaluated.

Results: The predictive nomogram had 8 predictor variables for prolonged PACU LOS, including age, ASA grade, active smoker, gastrointestinal disease, liver disease, and cardiovascular disease. This model displayed efficient calibration and moderate discrimination with a $C$-index of 0.662 ( $95 \%$ confidence interval, 0.603 to 0.721 ) for the training set, and 0.609 ( $95 \%$ confidence interval, 0.549 to 0.669 ) for the test set. Decision curve analysis demonstrated that the prolonged PACU LOS nomogram was reliable for clinical application when an intervention was decided at the possible threshold of $7 \%$.

Conclusions: We developed and validated a predictive nomogram with efficient calibration and moderate discrimination, and can be applied to identify patients most likely to be subjected to prolonged PACU LOS. This novel tool may shun overcrowding in PACU and optimize medical resource utilization.

\section{Introduction}

Laparoscopic Cholecystectomy (LC) is among the most commonly performed procedures in general surgery [1] [2]. Compared with open cholecystectomy (OC), LC is highly preferred as it is associated with less blood loss, less pain, and confer better cosmetic outcomes [3] [4] [5] [6]. Of note, reports from many studies have indicated a reduction of mortality and wound infection rates as well as improved quality of life after LC [7] [8] [9] [10]. The postanesthesia care unit (PACU) is an integral component of the perioperative workflow. PACU length of stay (LOS) impacts on operating room (OR) efficiency and present health burden to patients [11] [12] [13]. Extended PACU LOS can result in PACU backlog, decelerating the surgical schedule, this implies a reduced quality of care for other surgical patients [13]. Further, overcrowding in the PACU and prolonged PACU LOS may lead to poor clinical outcomes [11] [12] [14] [15].

Predictors for prolonged PACU LOS such as specific anesthesia type, surgical specialty, scheduled surgery duration, postoperative opioid administration, morbid obesity, hypertension, obstructive sleep apnoea have been described in different populations [16] [17] [18] [19] [20] [21] [22]. The studies in most cases assessed outpatients [16] [21], pediatric populations [17] [18] [19], or all adult surgical patients 
undergoing general anesthesia or regional anesthesia [20] [22]. However, no existing literature has reported a nomogram for prolonged PACU LOS of LC patients. Therefore, developing a predictive tool can be valuable in determining LC patients who require prolonged PACU LOS.

In this study, we collected data from a single tertiary-care medical institution in China and constructed a predictive nomogram. We purposed to provide a convenient predictive tool to anesthesiologists, nurses, or other care providers in PACU, to aid in the identification of patients requiring prolonged PACU LOS. This improves OR efficiency thereby minimizing healthcare burden.

\section{Methods}

\section{Study sample}

This study protocol was approved by Sun Yat-sen Memorial Hospital of Sun Yat-sen University Ethics Committee (approval number: SYSEC-KY-KS-2020-104). The resulting dataset remained unidentified and did not contain sensitive health information of patients as defined by the Human Research Protections Program in our institution, therefore, informed consent requirement by our Institutional Review Board was waved. For this retrospective analysis, two separate anesthesiologists from the Sun Yat-sen Memorial Hospital of Sun Yat-sen University manually obtained data from the electronic medical record system. All data from patients undergoing LC between January 1, 2018 and December 31, 2019 were extracted. This manuscript adheres to the applicable Equator guidelines for quality improvement studies.

Patients aged above 18 years, scheduled for LC, were included in the study dataset. We only chose patients who had received general anesthesia (i.e. balance anesthesia) as our institution did not administer neuraxial anesthesia (spinal or epidural) and combine with regional analgesia (Transversus Abdominis Plane blocks) to LC patients. Anesthesiologist selected endotracheal intubation (ETI) or laryngeal mask airway (LMA) as an airway management tool, based on personal preference. During the study period, the staffing and discharge criteria of the PACU remained unchanged in our hospital. All surgeries were performed by highly experienced surgeons using a standard procedure. In this study, we used short-acting opioids (e.g. remifentanil) and nonsteroidal anti-inflammatory drugs (NSAIDs, e.g. flurbiprofen axetil) to treat intraoperative and postoperative pain. We defined PACU LOS as the duration between admission to discharge from PACU, whereas prolonged PACU LOS was defined as time greater than or equal to the 75th percentile in each cohort, as described by previous studies [21]. Thus, we treated prolonged PACU LOS as a binary outcome rather than as continuous variables. Data of each patient derived from the electronic medical record system included: minutes in the PACU, sex, age (dichotomized to $\geq 65$ vs. $<65$ years of age to account for geriatric patients), body mass index (BMI) (dichotomized to $\geq 28$ vs. $<28 \mathrm{~kg} / \mathrm{m}^{2}$ to account for obesity in Chinese [23]), American Society of Anesthesiologists (ASA) grade, airway management device (ETI vs. LMA), surgery duration (dichotomized to $<60 \mathrm{vs.} \geq 60 \mathrm{~min}$ ). Besides, information on comorbidities including, diabetes mellitus, hypertension, active smoker, alcohol abuse, gastrointestinal disease, liver disease, cardiovascular disease, asthma, and allergic history was obtained. All the above data were considered as categorical variables. Of note, patients who were directly 
admitted to an intensive care unit, completely bypassed the PACU, Converted from Laparoscopic to Open Cholecystectomy during surgery, or missed values of any covariates were excluded in the analysis.

\section{Statistical analysis}

R software (R version 4.0.0) for statistical computing was used to perform all statistical analyses. After examining the data, patients with missing information were excluded before the model was constructed. This was flowed by categorizing the data set into a training set (cases during 2018) and a test set (cases during 2019).

Using the least absolute shrinkage and selection operator (LASSO) regression methods, the optimal predictive features were selected [24] [25]. Then, features with nonzero coefficients in the LASSO regression were selected to construct a predicting model, as we incorporated the multivariable logistic regression analysis. These features represented the odds ratio (OR) having a 95\% confidence interval (CI) and as P-value. All statistical significance levels were two-sided. Although there was no statistical significance in the training set, sex and BMI were still included in this model, as it has been the case with previous studies [12] [19] [21]. The nomogram was built to provide a convenient tool to predict probability of prolonged PACU LOS for each LC patients.

Calibration curves, in combination with the Hosmer-Lemeshow test [26], were plotted to evaluate the calibration of this predictive nomogram. Harrell's $C$-index was calculated to assess the discrimination performance of our nomogram [27]. Besides, the performance of the nomogram was determined using the test data set. We employed the Decision curve analysis (DCA) to assess the clinical application value of the prolonged PACU LOS risk nomogram [28]. In the training data set, quantification of the net benefits was undertaken at different threshold probabilities.

\section{Results}

\section{Clinical characteristics}

Among the 1,375 patients who underwent LC between January 1, 2018 and December 31, 2019 from our institution. However, due to the missing data of any of the covariates, 462 patients were excluded. A final total of 913 were included for the analyses after meeting the inclusion criteria, where, 456 and 457 patients were grouped in the training set and test set, respectively. All the characteristics of patients in each data set are highlighted in Table 1.

In the training set, the mean (standard deviation) and median (25\%-75\% interquartile range) PACU LOS were 57.13 minutes (20.87 minutes) and 55 minutes (44-66 minutes), respectively. In the test set, the mean (standard deviation) and median (25\%-75\% interquartile range) PACU LOS were 59.04 minutes (22.57 minutes) and 55 minutes (45-72 minutes), respectively. Therefore, considering our previous description, we defined prolonged PACU LOS as greater than 66 minutes in the training set and 72 minutes in the test set. 


\section{Feature selection}

Out of the clinical features, 15 features were reduced to six potential predictors based on 913 patients in the cohort (Fig. 1A and B). These predictors were featured with non-zero coefficients in the LASSO regression model.

The features included age, ASA grade, active smoker, gastrointestinal disease, liver disease, and cardiovascular disease (Table 2). Based on the previous studies, we also included sex and BMI in the model [12] [19] [21].

\section{Developing an individualized prediction model}

Following the logistic regression analysis, the results obtained are as shown in Table 2, which included sex, age, BMI, ASA grade, active smoker, gastrointestinal disease, liver disease, and cardiovascular disease. We developed a model in which the above independent predictors were incorporated and was presented as the nomogram (Fig. 2).

\section{Apparent performance and validation of the prolonged PACU LOS nomogram}

The calibration curve of the prolonged PACU LOS nomogram revealed reliable consistency between prediction and observation in the training set (Fig. 3). The $C$-index for the predictive nomogram was 0.662 ( $95 \% \mathrm{Cl}, 0.603$ to 0.721 ) for the training set whereas, in the test set, the $C$-index of the nomogram for prediction was 0.609 (95\% Cl, 0.549 to 0.669$)$. In the prolonged PACU LOS nomogram, apparent performance addressed a certain prediction potential.

\section{Clinical use}

The DCA for the prolonged PACU LOS nomogram showed that if the threshold probability of a patient and a doctor is $>7$ and $<52 \%$, respectively, using this nomogram to predict prolonged PACU LOS risk is highly beneficial compared to either the intervention-all-patients scheme or the intervention-none-patients scheme (Fig. 4). Within this range, the net benefit was comparable with several overlaps, based on the prolonged PACU LOS nomogram.

\section{Discussion}

Here we developed and validated a predictive nomogram for prolonged PACU LOS for LC patients. The predictors including sex, age, BMI, ASA grade, active smoker, gastrointestinal disease, liver disease, and cardiovascular disease were incorporated in our model. Usually, extended time spent in the PACU is both onerous to the patients and costly to the institution [11] [12] [29]. The nomogram suggested that younger (Age $<65)$ male patients, with obesity $\left(B M I>28 \mathrm{~kg} / \mathrm{m}^{2}\right)$, ASA grade $\otimes$, does not smoke, has no gastrointestinal disease, cardiovascular disease, or liver disease are $10 \%$ less likely to undergo prolonged PACU LOS. In this study, we provide a novel prediction nomogram vital in helping care providers (anesthesiologists, nurses) in PACU to identify patients with higher chances of being subjected to 
prolonged PACU LOS. Within institutions such as community hospitals and clinics with fewer ORs, this predictive tool is highly suitable and beneficial. It is worth noting that all predictors included in this model can be determined preoperatively.

Previously, several studies have revealed risk factors for prolonged PACU LOS in various surgical populations [16] [17] [18] [19] [20] [21] [22]. These studies suggest the need to focus on a special subset of patients as a way of reducing research bias [21], rather than focusing on all patients scheduled for surgery with general anesthesia [20]. To the best of our knowledge, so far, no study has investigated the application of a nomogram to predict prolonged PACU LOS of LC patients.

Consistent with previous studies, we revealed that age is associated with prolonged PACU LOS [19] [20]. The geriatric population may be associated with many postoperative complications and poor drug metabolism, in this case, longer recovery time is required after the operation. Likewise, patients with higher ASA grades are more likely to undergo prolonged PACU LOS. Also, we found that active smoking may result in longer PACU duration, which may be associated with a higher risk of respiratory complications. Elsewhere, a study by Coccolini et al. [8] demonstrated that LC had decreased odds for respiratory complications (such as airway obstruction and pneumonia) when compared with OC. Interestingly, we observed that patients with gastrointestinal diseases, such as chronic gastritis, gastric and duodenal ulcers, showed increased odds for prolonged PACU LOS. This is because such patients, in most cases, have electrolyte disorders, anemia, and high risk of postoperative nausea and vomiting (PONV), they should be carefully monitored, thus require longer stay in PACU [30]. Due to the postoperative cardiovascular complications, it can strongly be suggested that cardiovascular disease is associated with prolonged PACU LOS.

On the contrary, patients who preoperatively had liver disease may be at a lower risk of extended time in PACU. This is closely linked to the use of anesthetic and anesthesia management of LC. For example, when handling the surgery patients with liver disease, drugs that are not metabolized by the liver (e.g. cisatracurium, remifentanil, and sevoflurane) are administered while the dosage of an anesthetic is reduced to protect liver function. Also, inconsistent with previous findings [19] [21], we found that higher BMI may result in a lower probability of prolonged PACU LOS. Based on our institution practice, we routinely selected short-acting anesthetics to induce general anesthesia and timely stopped drug infusion among obesity patients. A previous study revealed that the airway management device (i.e. LMA) was associated with prolonged PACU LOS [31], however, no statistical difference was found in this study. Moreover, Abdi et al. [32] reported that LMA, with less pharyngolaryngeal discomfort and pain, showed low risks for prolonged PACU LOS compared to ETI.

This study had several limitations. First, the retrospective design of this study implies that the selection bias and information bias is unavoidable. Second, the accuracy of the analysis relies on data records, storage, and retrieval from the medical system. Some factors include nonclinical and social issues, and difficult to extract from our electronic records. Therefore, other factors that may be associated with prolonged PACU LOS exist, and their exclusion may affect the discriminating ability in our model. Third, 
we included patients with specific surgery and anesthesia type, thus, the generalizability was uncertain for other patients with different types of surgical procedures and anesthesia. Finally, we report on a single-institution experience, therefore, this predictive nomogram for patients undergoing LC need to be further evaluated in the other institution and countries.

\section{Conclusion}

We developed a predictive nomogram with a relatively moderate accuracy to aid in identifying which LC patients are more likely to be subjected to prolonged PACU LOS. These predictors may contribute to prior planning in PACU for bed space and allow for optimization of the OR efficiency.

\section{Abbreviations}

LC: laparoscopic cholecystectomy; PACU: postanesthesia care unit; LOS: length of stay; OC: open cholecystectomy; OR: operating room; ETI: endotracheal intubation; LMA: laryngeal mask airway; BMI: body mass index; ASA: American Society of Anesthesiologists; LASSO: least absolute shrinkage and selection operator; OR: odds ratio; Cl: confidence interval; DCA: decision curve analysis; PONV: postoperative nausea and vomiting

\section{Declarations}

\section{Acknowledgments}

We appreciate Qiaoyun Li, department of Physiology, Zhongshan School of Medicine, Sun Yat-Sen University in Guangzhou, for her valuable comments on the manuscript.

\section{Authors' contributions}

Study conception and design [BBC, LL, WBG]; Methodology: [BBC, WBG, XFS]; Formal analysis and investigation: [BBC, XFS]; Writing-original draft preparation: [LL, XFS]; Writing-review and editing: [BBC, LL, JFZ]; Funding acquisition: [JFZ]; Resources: [BBC, LL]; Supervision: [JFZ, WBG].

\section{Funding}

This work was supported by the Beijing Medical Award Foundation (NO. YXJL-2019-0163-0019).

\section{Availability of data and materials}

The data that support the findings of this study are available from the corresponding author upon request.

\section{Ethics approval}


This study was performed in line with the principles of the Declaration of Helsinki. The study protocol was approved by Sun Yat-sen Memorial Hospital of Sun Yat-sen University Ethics Committee (approval number: SYSEC-KY-KS-2020-104).

\section{Informed Consent}

The resulting dataset remained unidentified and did not contain sensitive health information of patients as defined by the Human Research Protections Program in our institution, therefore, informed consent requirement by our Institutional Review Board was waved.

\section{Consent for publication}

Not applicable.

\section{Competing interests}

Not applicable.

\section{References}

1. Csikesz N, Ricciardi R, Tseng JF, Shah SA. Current status of surgical management of acute cholecystitis in the United States. World J Surg. 2008;32:2230-6.

2. Mou D, Tesfasilassie T, Hirji S, Ashley SW. Advances in the management of acute cholecystitis. Ann Gastroenterol Surg. 2019;3(3):247-253.

3. Finan KR, Leeth RR, Whitley BM, Klapow JC, Hawn MT. Improvement in gastrointestinal symptoms and quality of life after cholecystectomy. Am J Surg. 2006;192:196-202.

4. Al Masri S, Shaib Y, Edelbi M, et al. Predicting Conversion from Laparoscopic to Open Cholecystectomy: A Single Institution Retrospective Study. World J Surg. 2018;42:2373-82.

5. Rafiq MS, Khan MM. Scar Pain, Cosmesis and Patient Satisfaction in Laparoscopic and Open Cholecystectomy. J Coll Physicians Surg Pak. 2016;26:216-9.

6. Bisgaard T. Analgesic treatment after laparoscopic cholecystectomy: a critical assessment of the evidence. Anesthesiology. 2006;104:835-46.

7. Zarin M, Khan MA, Khan MA, Shah SAM. Critical view of safety faster and safer technique during laparoscopic cholecystectomy? Pak J Med Sci Q. 2018;34:574-7.

8. Coccolini F, Catena F, Pisano M, et al. Open versus laparoscopic cholecystectomy in acute cholecystitis. Systematic review and meta-analysis. Int J Surg. 2015;18:196-204.

9. Quintana JM, Cabriada J, Aróstegui I, et al. Health-related quality of life and appropriateness of cholecystectomy. Ann Surg. 2005;241:110-8.

10. Wolfe BM, Gardiner B, Frey CF. Laparoscopic Cholecystectomy: A Remarkable Development. JAMA. 2015;314:1406. 
11. Ehrenfeld JM, Dexter F, Rothman BS, et al. Lack of utility of a decision support system to mitigate delays in admission from the operating room to the postanesthesia care unit. Anesth Analg. 2013;117:1444-52.

12. Waddle JP, Evers AS, Piccirillo JF. Postanesthesia care unit length of stay: quantifying and assessing dependent factors. Anesth Analg. 1998;87:628-33.

13. Weissman C. The enhanced postoperative care system. J Clin Anesth. 2005;17:314-22.

14. Weissman C, Scemama J, Weiss YG. The ratio of PACU length-of-stay to surgical duration: Practical observations. Acta Anaesthesiol Scand. 2019;63:1143-51.

15. Mann-Farrar J, Egan E, Higgins A, et al. Are Postoperative Clinical Outcomes Influenced by Length of Stay in the Postanesthesia Care Unit? J Perianesth Nurs. 2019;34:386-93.

16. Elsharydah A, Walters DR, Somasundaram A, et al. A preoperative predictive model for prolonged post-anaesthesia care unit stay after outpatient surgeries. J Perioper Pract. 2020;30:91-6.

17. Anandan D, Zhao S, Whigham AS. Factors Affecting Post-Anesthesia Care Unit Length of Stay in Pediatric Patients after an Adenotonsillectomy. Ann Otol Rhinol Laryngol. 2020:3489420931557.

18. Silins V, Julien F, Brasher C, et al. Predictive factors of PACU stay after herniorraphy in infant: a classification and regression tree analysis. Paediatr Anaesth. 2012;22:230-8.

19. Walia H, Balaban O, Jacklen M, et al. Pilot study comparing post-anesthesia care unit length of stay in moderately and severely obese children. J Anesth. 2017;31:510-6.

20. Seago JA, Weitz S, Walczak S. Factors influencing stay in the postanesthesia care unit: a prospective analysis. J Clin Anesth. 1998;10:579-87.

21. Gabriel RA, Waterman RS, Kim J, Ohno-Machado L. A Predictive Model for Extended Postanesthesia Care Unit Length of Stay in Outpatient Surgeries. Anesth Analg. 2017;124:1529-36.

22. Corey JM, Bulka CM, Ehrenfeld JM. Is regional anesthesia associated with reduced PACU length of stay?: A retrospective analysis from a tertiary medical center. Clin Orthop Relat Res. 2014;472:142733.

23. Xia M, Liu S, Ji N, et al. BMI $35 \mathrm{~kg} / \mathrm{m}^{2}$ does not fit everyone: a modified STOP-Bang questionnaire for sleep apnea screening in the Chinese population. Sleep Breath. 2018;22:1075-82.

24. Sauerbrei W, Royston P, Binder H. Selection of important variables and determination of functional form for continuous predictors in multivariable model building. Stat Med. 2007;26:5512-28.

25. Huang YQ, Liang CH, He L, et al. Development and Validation of a Radiomics Nomogram for Preoperative Prediction of Lymph Node Metastasis in Colorectal Cancer. J Clin Oncol. 2016;34:215764.

26. Kramer AA, Zimmerman JE. Assessing the calibration of mortality benchmarks in critical care: The Hosmer-Lemeshow test revisited. Crit Care Med. 2007;35:2052-6.

27. Pencina MJ, D'Agostino RB. Overall $C$ as a measure of discrimination in survival analysis: model specific population value and confidence interval estimation. Stat Med. 2004;23:2109-23. 
28. Vickers AJ, Cronin AM, Elkin EB, Gonen M. Extensions to decision curve analysis, a novel method for evaluating diagnostic tests, prediction models and molecular markers. BMC Med Inform Decis Mak. 2008;8:53.

29. Dabu-Bondoc S, Vadivelu N, Shimono C, et al. Intravenous dextrose administration reduces postoperative antiemetic rescue treatment requirements and postanesthesia care unit length of stay. Anesth Analg. 2013;117:591-6.

30. Kim GH, Lee HD, Kim M, et al. Fate of dyspeptic or colonic symptoms after laparoscopic cholecystectomy. J Neurogastroenterol. 2014;20:253-60

31. Tian Y, Wu XY, Li L, Ma L, Li YF. A clinical trial evaluating the laryngeal mask airway-Supreme in obese children during general anesthesia. Arch Med Sci. 2017;13:183-90.

32. Abdi W, Amathieu R, Adhoum A, et al. Sparing the larynx during gynecological laparoscopy: a randomized trial comparing the LMA Supreme and the ETT. Acta Anaesth Scand. 2010;54:141-6.

\section{Tables}

Table 1. Clinical Characteristics in the Training Versus Test Data Sets. 


\begin{tabular}{|c|c|c|}
\hline & $\begin{array}{l}\text { Training Data Set } \\
N(\%)\end{array}$ & $\begin{array}{l}\text { Test Data Set } \\
N(\%)\end{array}$ \\
\hline Total & 456 & 457 \\
\hline Median PACU LOS (25\%, 75\% IQR) & $55(44,66)$ & $55(34,72)$ \\
\hline Prolonged PACU LOS & 113(24.78) & 114(24.95) \\
\hline Age $\geq 65$-years old & 122(26.75) & 107(23.41) \\
\hline Male sex & $222(48.68)$ & $213(46.61 \%)$ \\
\hline $\mathrm{BMI} \geq 28 \mathrm{~kg} / \mathrm{m}^{2}$ & $49(10.75)$ & $39(8.53)$ \\
\hline \multicolumn{3}{|l|}{ ASA Physical Status } \\
\hline$\nabla$ & $29(6.36)$ & $29(6.35)$ \\
\hline$\nabla$ & $326(71.49)$ & $337(73.74 \%)$ \\
\hline$\otimes$ & $97(21.27)$ & $90(19.69 \%)$ \\
\hline ૫ & $4(0.88)$ & $1(0.22)$ \\
\hline \multicolumn{3}{|l|}{ Airway management device } \\
\hline ETI & 382(83.77) & 296(64.77) \\
\hline LMA & $74(16.23)$ & 161(35.23) \\
\hline \multicolumn{3}{|l|}{ Surgery duration (min) } \\
\hline$<60$ & $141(30.9)$ & $143(31.3)$ \\
\hline$\geq 60$ & $315(69.1)$ & $314(68.7)$ \\
\hline \multicolumn{3}{|l|}{ Comorbidities } \\
\hline Diabetes melitus & 41(8.99) & $44(9.63)$ \\
\hline Hypertension & $93(20.39)$ & $98(21.44)$ \\
\hline Cardiovascular disease & $24(5.26)$ & 17(3.72) \\
\hline Liver disease & $39(8.55)$ & $30(6.56)$ \\
\hline Gastrointestinal disease & 17(3.73) & $4(0.66)$ \\
\hline Active smoker & $58(12.72)$ & $35(7.66)$ \\
\hline Asthma & $5(1.10)$ & $3(0.66)$ \\
\hline Alcohol abuse & $19(4.17)$ & $3(0.66)$ \\
\hline Allergic history & $48(10.53)$ & 41(8.97) \\
\hline
\end{tabular}


Prolonged PACU LOS was defined as time greater than or equal to the 75th percentile in each cohort.

PACU postanesthesia care unit, LOS length of stay, IQR interquartile range, $B M /$ body mass index, $A S A$ American Society of Anesthesiologists, ETI endotracheal intubation, LMA laryngeal mask airway.

Table 2 Prediction Factors for Prolonged PACU LOS Built on the Training Set.

\begin{tabular}{|llll|}
\hline & $\boldsymbol{\beta}$ & OR (95\% Cl) & $\boldsymbol{P}$ \\
\hline Intercept & -2.241 & $0.106(0.029-0.368)$ & $<.001$ \\
\hline Sex & 0.058 & $1.059(0.650-1.736)$ & .818 \\
\hline Age & 0.757 & $2.131(1.269-3.571)$ & .004 \\
\hline BMI & -0.395 & $0.673(0.299-1.395)$ & .310 \\
\hline ASA grade & 0.318 & $1.374(0.863-2.195)$ & .181 \\
\hline Active smoker & 0.739 & $2.093(1.067-4.057)$ & .029 \\
\hline Gastrointestinal disease & 1.142 & $3.134(1.122-8.629)$ & .026 \\
\hline Liver disease & -0.719 & $0.487(0.173-1.172)$ & .135 \\
\hline Cardiovascular disease & 0.637 & $1.891(0.756-4.669)$ & .166 \\
\hline
\end{tabular}

$\beta$ is the regression coefficient.

PACU postanesthesia care unit, LOS length of stay, BMI body mass index, ASA American Society of Anesthesiologists, $O R$ odds ration, $\mathrm{Cl}$ confidence interval.

\section{Figures}



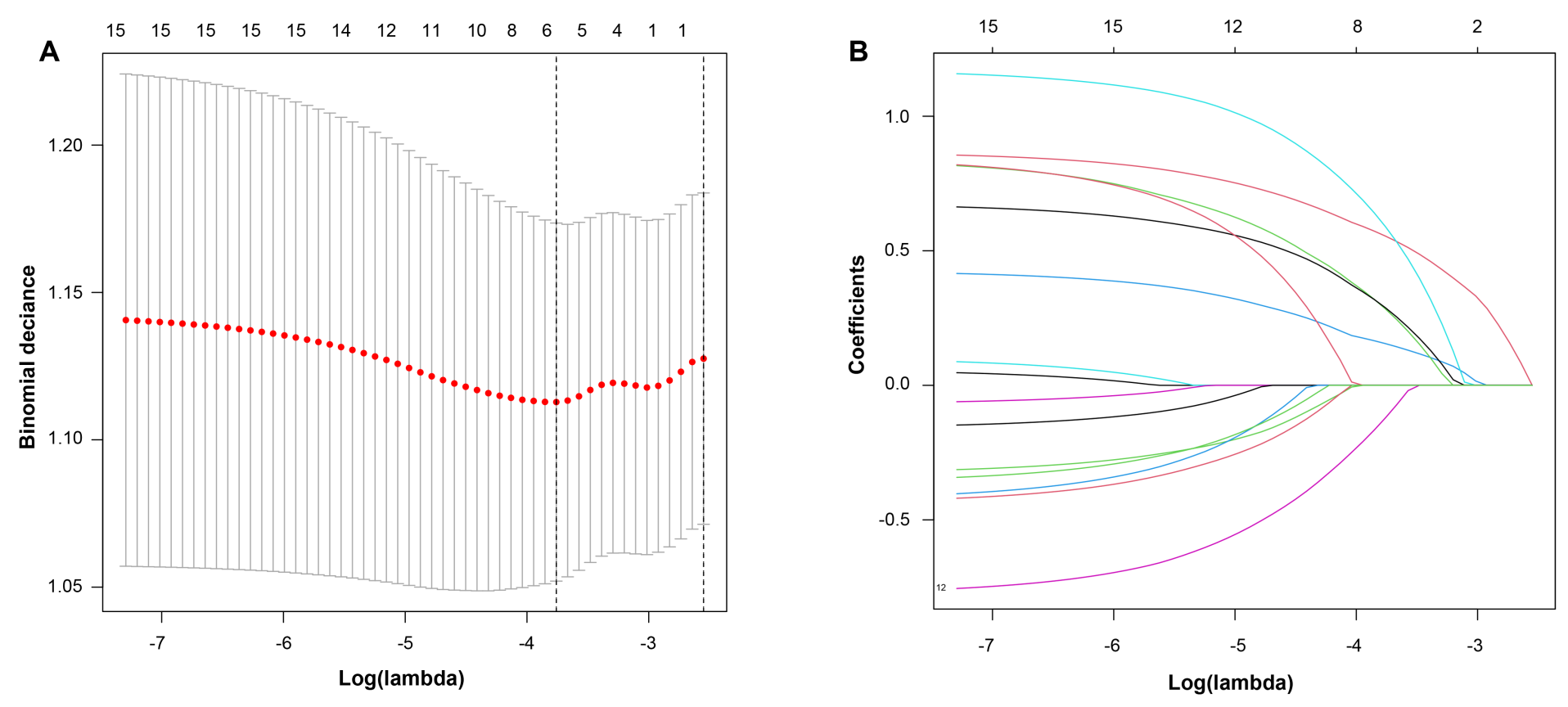

Figure 1

Patient feature selection using the LASSO regression model. A, Optimal parameter (lambda) selection. B, LASSO coefficient profiles of the 15 texture features. Abbreviations: LASSO, least absolute shrinkage and selection operator. 


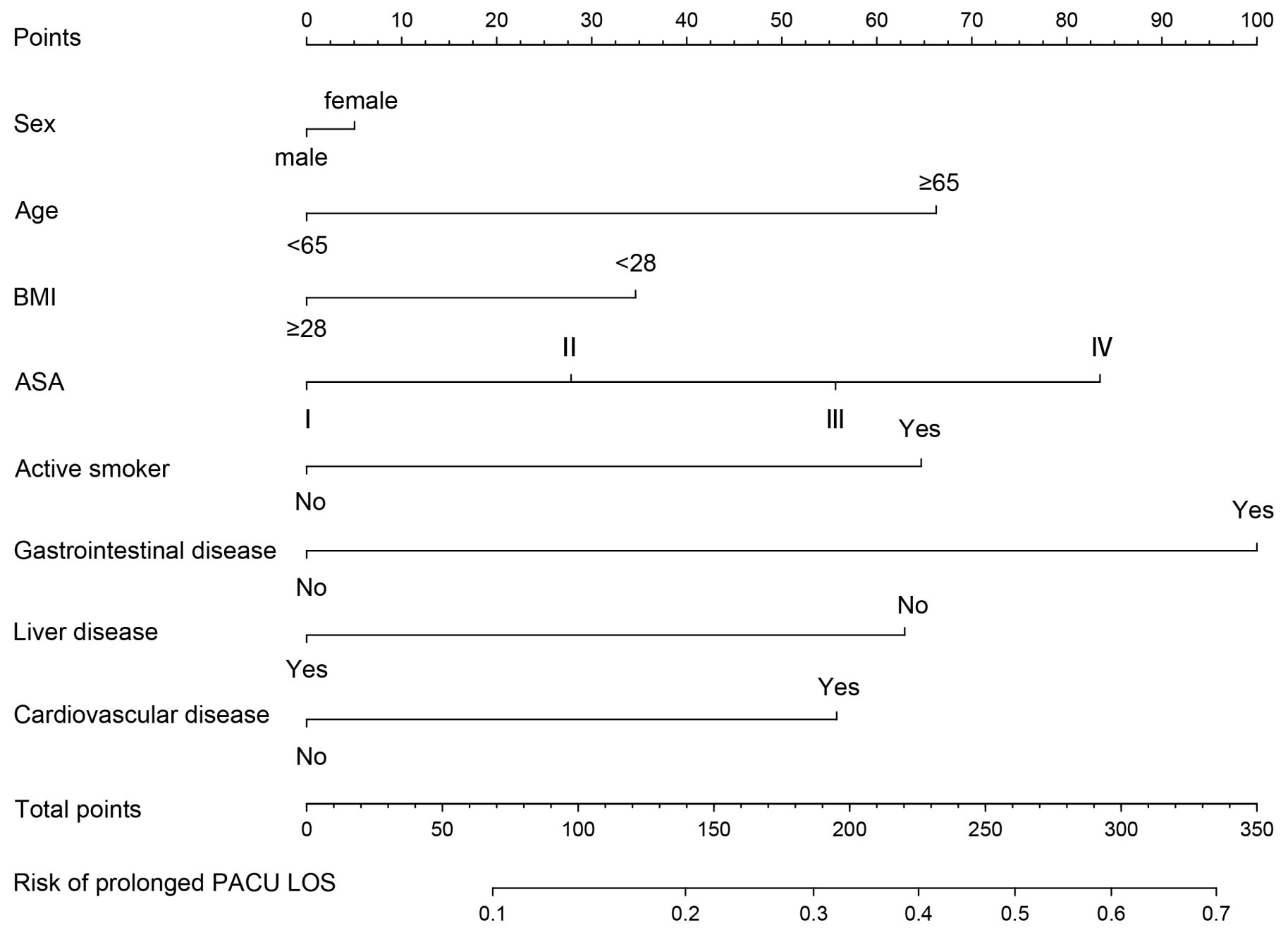

\section{Figure 2}

Developed prolonged PACU LOS nomogram. This nomogram was developed on the training set, with the sex, age, BMI, ASA grade, active smoker, gastrointestinal disease, liver disease and cardiovascular disease. Abbreviations: BMI, body mass index; ASA, American Society of Anesthesiologists; PACU, postanesthesia care unit; LOS, length of stay. 


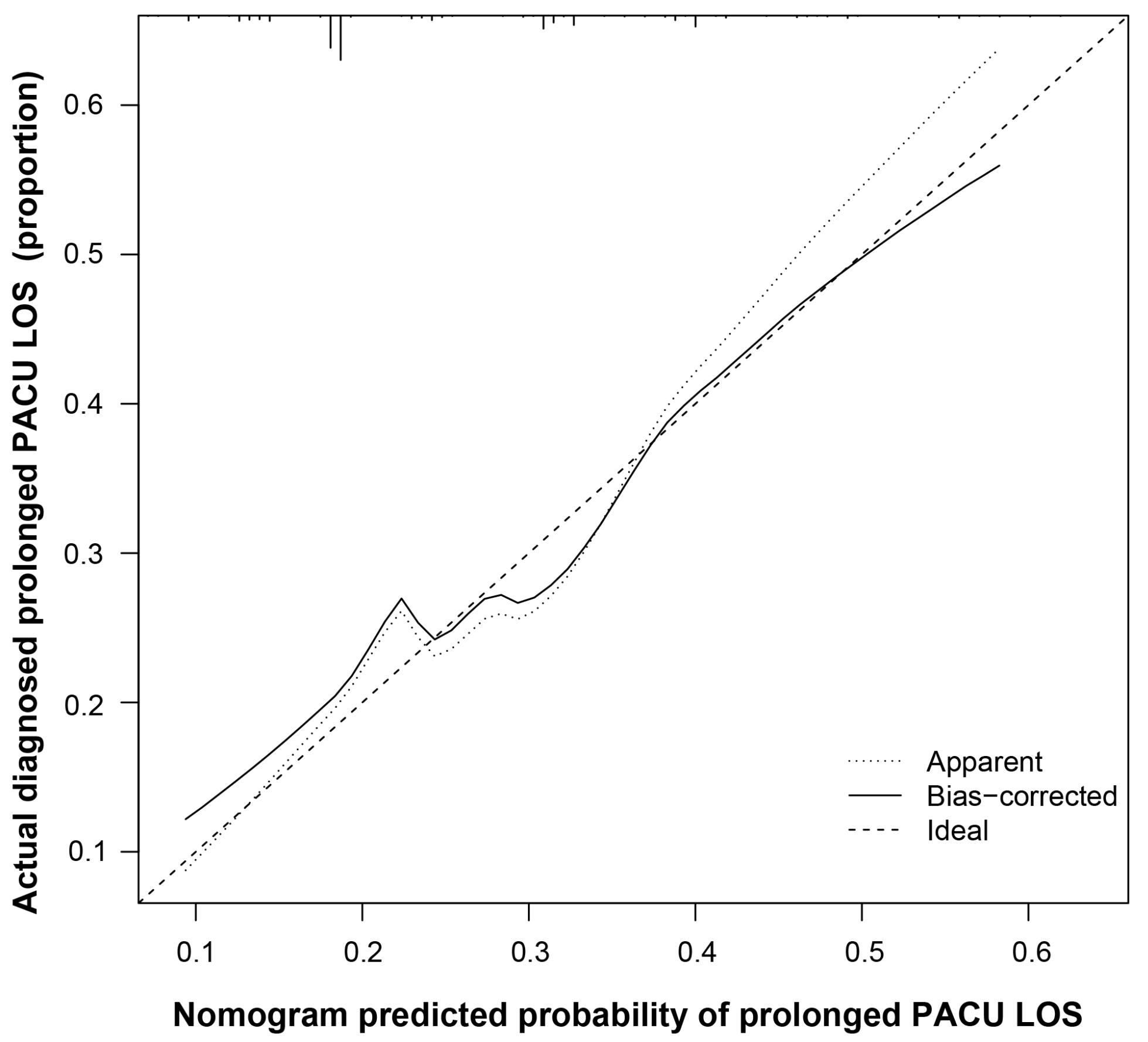

Figure 3

Calibration curves of the prolonged PACU LOS nomogram prediction on the training set. The $x$-axis represents the predicted prolonged PACU LOS risk. The $y$-axis represents the actual diagnosed prolonged PACU LOS. 


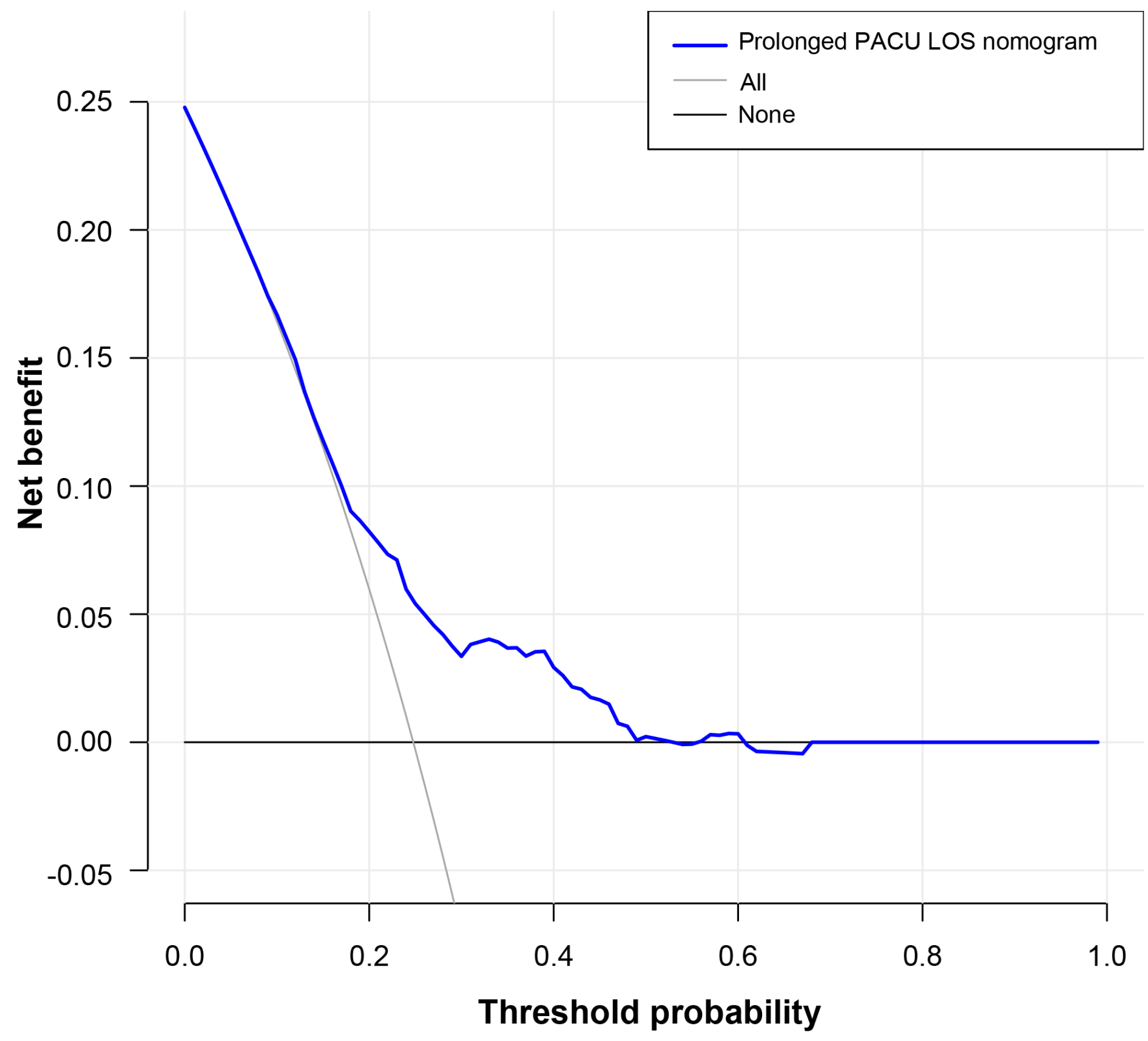

Figure 4

Decision curve analysis for the prolonged PACU LOS nomogram. 